\title{
A Minimally Destructive Protocol for DNA Extraction from Ancient Teeth
}

Éadaoin Harney ${ }^{1,2,3,4,{ }^{*}, a}$, Olivia Cheronet ${ }^{5,{ }^{*}, a}$, Daniel M. Fernandes ${ }^{5,6}$, Kendra Sirak $^{3,4}$, Matthew Mah $^{3,7}$, Rebecca Bernardos ${ }^{3,8}$, Nicole Adamski ${ }^{3,8}$, Nasreen Broomandkhoshbacht ${ }^{3,8}$, Kimberly Callan $^{3,8}$, Ann Marie Lawson ${ }^{3,8}$, Jonas Oppenheimer ${ }^{3,8}$, Kristin Stewardson ${ }^{3,8}$, Fatma Zalzala ${ }^{3,8}$, Alexandra Anders ${ }^{9}$, Francesca Candilio $^{10}$, Mihai Constantinescu ${ }^{11}$, Alfredo Coppa ${ }^{3,5,12}$, Ion Ciobanu $^{13}$, János Dani ${ }^{14}$, Zsolt Gallina ${ }^{15}$, Francesco Genchi ${ }^{12}$, Emese Gyöngyvér Nagy ${ }^{14}$, Tamás Hajdu ${ }^{16,17}$, Magdolna Hellebrandt ${ }^{18}$, Antónia Horváth ${ }^{18}$, Ágnes Király ${ }^{19}$, Krisztián Kiss ${ }^{16,17}$, Barbara Kolozsi ${ }^{14}$, Péter Kovács ${ }^{20}$, Kitti Köhler ${ }^{19}$, Michaela Lucci ${ }^{21}$, Ildikó Pap ${ }^{17}$, Sergiu Popovici'22, Pál Raczky9 ${ }^{9}$, Angela Simalcsik ${ }^{23,24}$, Tamás Szeniczey ${ }^{16,17}$, Sergey Vasilyev ${ }^{25,26}$, Cristian Virag ${ }^{27}$, Nadin Rohland ${ }^{3,7}$, David Reich ${ }^{2,3,4,7,8}$, Ron Pinhasi ${ }^{4, a}$

${ }^{1}$ Dept. of Organismic and Evolutionary Biology, Harvard University, Cambridge, MA, 02138, USA

${ }^{2}$ The Max Planck-Harvard Research Center for the Archaeoscience of the Ancient Mediterranean, Cambridge, MA, 02138, USA and Jena, D-07745, Germany

${ }^{3}$ Dept. of Genetics, Harvard Medical School, Boston, MA, 02115, USA

${ }^{4}$ Department of Human Evolutionary Biology, Harvard University, Cambridge, MA 02138, USA

${ }^{5}$ Dept. of Evolutionary Anthropology, University of Vienna, Vienna, 1090, Austria

${ }^{6}$ CIAS, Department of Life Sciences, University of Coimbra, 3000-456 Coimbra, Portugal

${ }^{7}$ Broad Institute of Harvard and MIT, Cambridge, MA 02142 USA

${ }^{8}$ Howard Hughes Medical Institute, Harvard Medical School, Boston, MA, 02115, USA

${ }^{9}$ Institute of Archaeology, Eötvös Loránd University, Budapest, Hungary

${ }^{10}$ Superintendency of Archaeology, Fine Arts and Landscape for the city of Cagliari and the provinces of Oristano and South Sardinia, Cagliari, Italy

${ }^{11} \mathrm{Fr}$. I. Rainer Institute of Anthropology, Bucharest, 050711, Romania

${ }^{12}$ Department of Environmental Biology, Sapienza University, Rome, Italy

${ }^{13}$ National Archaeological Agency, Institute of Bioarchaeological and Ethnocultural Research, Chisinau, Republic of Moldova

${ }^{14}$ Déri Museum, 4026 Debrecen, Déri tér 1, Hungary

${ }^{15}$ Ásatárs Kulturális, Régészeti Szolgáltató és Kereskedelmi Ltd. Hungary

${ }^{16}$ Department of Biological Anthropology, Eötvös Loránd University, Budapest, Hungary

${ }^{17}$ Department of Anthropology, Hungarian Natural History Museum, Budapest, Hungary

${ }^{18}$ Herman Ottó Museum, 3529 Miskolc, Görgey Artúr u. 28, Hungary

${ }^{19}$ Institute of Archaeology, Research Centre for the Humanities, Budapest, Hungary

${ }^{20}$ Damjanich János Museum, Szolnok, Kossuth tér 4, H- 5000, Hungary

${ }^{21}$ Department of History, Anthropology, Religion, Arts and Performing Arts, Sapienza University, Rome, Italy

${ }^{22}$ National Agency for Archaeology, Chișinău, Republic of Moldova

${ }^{23}$ Olga Necrasov Center for Anthropological Research, Romanian Academy, lasi, Romania

${ }^{24}$ Institute of Bioarchaeological and Ethnocultural Research, Chisinau, Republic of Moldova

${ }^{25}$ Institute of Ethnology and Anthropology of the Russian Academy of Sciences, 119991 Moscow, Russia

${ }^{26}$ Center for Egyptological Studies of the Russian Academy of Sciences, 119071 Moscow, Russia

${ }^{27}$ Satu Mare County Museum, Satu Mare, Romania

*These authors contributed equally

${ }^{a}$ Correspondence to Ron Pinhasi (Tel: ron.pinhasi@univie.ac.at). Correspondence may also be addressed to

Éadaoin Harney (eadaoinharney@gmail.com) or Olivia Cheronet (olivia.cheronet@univie.ac.at)

Running Title: Minimally Destructive DNA Extraction from Ancient Teeth

Keywords: Ancient DNA, Teeth, Cementum, Minimally Destructive Sampling 


\section{$1 \quad$ ABSTRACT}

2 Ancient DNA sampling methods-although optimized for efficient DNA extraction-are

3 destructive, relying on drilling or cutting and powdering (parts of) bones and teeth. As the

4 field of ancient DNA has grown, so have concerns about the impact of destructive

5 sampling of the skeletal remains from which ancient DNA is obtained. Due to a particularly

6 high concentration of endogenous DNA, the cementum of tooth roots is often targeted for

7 ancient DNA sampling, but standard destructive sampling methods often result in the loss

8 of at least one entire root. Here, we present a minimally destructive method for extracting

9 ancient DNA from dental cementum present on the surface of tooth roots. This method

10 does not require destructive drilling or grinding, and, following extraction, the tooth

11 remains safe to handle and suitable for most morphological studies, as well as other

12 biochemical studies, such as radiocarbon dating. We extracted and sequenced ancient

13 DNA from 30 teeth (and 9 corresponding petrous bones) using this minimally destructive

14 extraction method in addition to a typical tooth sampling method. We find that the

15 minimally destructive method can provide ancient DNA that is of comparable quality to

16 extracts produced from teeth that have undergone destructive sampling processes.

17 Further, we find that a rigorous cleaning of the tooth surface combining diluted bleach

18 and UV light irradiation seems sufficient to minimize external contaminants usually

19 removed through the physical removal of a superficial layer when sampling through

20 regular powdering methods. 


\section{INTRODUCTION}

22 Over the past decade, the field of ancient DNA has experienced a rapid increase in the

23 number of ancient genomes published each year (Slatkin and Racimo 2016) as a

24 consequence of advances in ancient DNA sampling (Gamba et al. 2014; Damgaard et al.

25 2015), extraction (Dabney et al. 2013a; Rohland et al. 2018), and enrichment (Carpenter

26 et al. 2013; Fu et al. 2013) techniques. As our ability to sequence large numbers of ancient

27 individuals has increased, discussions about the destructive nature of ancient DNA

28 sampling — which typically requires drilling or cutting and powdering ancient bones and

29 teeth—have become more prominent (Makarewicz et al. 2017; Prendergast and Sawchuk

30 2018; Sirak and Sedig 2019). The identification of the osseous inner ear, and specifically

31 the cochlea (located in the petrous portion of the temporal bone), as an optimal source of

32 ancient DNA (Gamba et al. 2014; Pinhasi et al. 2015; Pinhasi et al. 2019) is one of the

33 driving factors in this revolution, making it possible to access ancient DNA from

34 geographic regions with climatic conditions unfavorable to ancient DNA preservation.

35 However, accessing this optimal source of ancient DNA results in the destruction of the

36 inner ear morphology, which is a valuable source of morphological information (de León

37 et al. 2018). While there are protocols that reduce the destructive nature of sampling, by

38 sampling from the ossicles of the inner ear (Sirak et al. 2020) or performing targeted

39 drilling of the cochlea through the cranial base of complete or reconstructed crania (Sirak

40 et al. 2017), some destruction (including that of morphologically-informative inner ear

41 components) is inevitable. As a consequence, this and other less-invasive methods may

42 be considered unsuitable in cases where samples are of particular anthropological value

43 and are subject to stringent restrictions on permissible sampling practices. 
45 Teeth are a valuable alternative to the sampling of the cochlea (Gamba et al. 2014;

46 Damgaard et al. 2015), especially because they are particularly numerous in osteological

47 collections, due to the fact that individuals have many more teeth than petrous bones and

48 to their resistance to taphonomic decomposition. Despite this, little has been published

49 outlining optimal practices for sampling from teeth. Traditionally, the standard practice

50 has been to grind or drill large chunks of the tooth root to a powder (Rohland and Hofreiter

51 2007), as the crown enamel is largely inorganic and is therefore unlikely to contain a

52 substantial amount of endogenous DNA (Higgins and Austin 2013). In an attempt to

53 minimize potential external contaminants, the surface layer is often removed to access

54 the "untouched" dentine and pulp. However, this practice removes some, if not all, of the

55 thin layer of cementum that coats the inferior portion of dental roots.

57 The cellular cementum is rich in cementocytes, which are DNA containing cells that

58 remain encased in the mineral structure of the tooth after death (Bosshardt and Selvig

59 1997). Cementum also shares several histological properties with the cochlear region of

60 the petrous that are thought to contribute to its high level of DNA preservation, including

61 similarities between cementocytes (Zhao et al. 2016) and osteocytes, which are

62 hypothesized to be serve as repositories of ancient DNA in bones (Bell et al. 2008; Pinhasi

63 et al. 2015). Like the cochlea, cementum also does not undergo remodeling (but, unlike

64 the cochlea, it continues to accumulate throughout life) and the haphazard organization

65 of collagen fibers in cementum resembles that of woven bone (Freeman 1994; Grzesik et

66 al. 2000). Assessment of DNA preservation in ancient teeth shows that dental cementum 
67 contains a substantially higher proportion of endogenous DNA than dentine from the

68 same tooth (Damgaard et al. 2015). Furthermore, in a direct comparison between

69 cementum and petrous samples, Hansen et al. (2017) find that cementum and petrous

70 yield a comparable amount of endogenous DNA in well-preserved samples, although in

71 poorly-preserved individuals, the petrous yields a higher proportion of endogenous

72 molecules. The only published method for sampling DNA from the cementum

73 recommends a targeted method for extracting DNA from teeth using an "inside-out"

74 approach that involves removing the crown and subsequently using a fine drill to remove

75 as much pulp and dentine as possible from the tooth root to ultimately obtain a "case" of

76 cementum (Damgaard et al. 2015). However, this valuable approach may still not be able

77 to perfectly isolate the extremely thin and brittle layer of cementum, which ranges from

$78 \quad 20-50 \mu \mathrm{m}$ thick at the cementoenamel junction, to $150-200 \mu \mathrm{m}$ thick at the apex of the

$79 \operatorname{root}($ Freeman 1994).

81 Here, we present an alternative, minimally destructive protocol for sampling ancient DNA

82 from tooth cementum that does not require drilling or cutting, thereby maintaining the

83 morphological integrity of the tooth. The technique isolates ancient DNA from the

84 cementum of tooth roots by directly exposing the outermost layer of a portion of the tooth

85 root to a lysis buffer for a short incubation period, following a non-destructive

86 decontamination procedure. Similar less destructive methods have been reported in

87 previous PCR-based mitochondrial ancient DNA studies (Rohland et al. 2004; Bolnick et

88 al. 2012) and in forensic contexts (Correa et al. 2019). However, the ancient DNA

89 obtained using these strategies was typically less well preserved and of a lesser quantity 
90 than DNA obtained using more destructive methods. Additionally, in some cases

91 (Rohland et al. 2004), the hazardous chemicals used during sampling may have

92 compromised safe handling and future chemical analyses of the remains. In this study,

93 we conduct a systematic evaluation of the application of a minimally destructive sampling

94 technique in a next generation sequencing context. This protocol is further optimized by

95 enabling targeted sampling from the very thin dental cementum layer, which increases

96 the quality of ancient DNA sampled from the tooth while giving researchers the ability to

97 fully preserve the dental crowns and all but the fine external detail of the roots. After

98 sampling, teeth can be safely handled and remain suitable for subsequent morphological

99 and biomolecular analyses, such as radiocarbon dating (Korlević et al. 2018).

\section{RESULTS}

101 We selected thirty ancient individuals (Table 1; Supplementary Table 1) for a comparative

102 analysis of the quality of ancient DNA-as measured through metrics such as the

103 proportion of endogenous molecules of shotgun data, sample complexity and

104 contamination rate-that could be obtained from an individual using this minimally

105 destructive extraction method versus standard sampling procedures that rely on cutting

106 and powdering tooth samples. From each individual we sampled a single multi-rooted

107 tooth, from which the roots were removed via cutting (note that the tooth roots were cut

108 in order to make it possible to process the samples using several independent methods,

109 but cutting is not required by the minimally destructive sampling protocol) and were each

110 randomly assigned to undergo one of the following extraction treatments. We extracted

111 ancient DNA from a tooth root that was processed using the minimally destructive 
112 extraction protocol described in this paper (Method "MDE"; for "Minimally Destructive

113 Extraction") and a second whole tooth root of the same tooth, that was completely

114 powdered via milling (Method "WTR"; for "Whole Tooth Root"). We also generated

115 extracts from powder produced from petrous bones for 10 of the same individuals using

116 the method described by Pinhasi et al. (2019) (Method "P"; for "Petrous"). In one case

117 (individual 3), we discovered through subsequent bioinformatic analyses that the petrous

118 bone and tooth sampled did not originate from the same individual, and we therefore

119 exclude the petrous bone results from further analyses. DNA preservation in two

120 individuals (5 and 6) was uniformly poor, with no more than 10,000 sequences aligning

121 to the 1.24 million sites captured through targeted enrichment (out of $\sim 5$ million unique

122 reads sequenced) from any of the libraries generated. Furthermore, all of these double-

123 stranded libraries exhibited C-to-T damage rates at the terminal ends of molecules of less

124 than 3\% - the recommended minimum threshold for assessing ancient DNA authenticity

125 in partially UDG treated libraries (Rohland et al. 2015). These samples are considered to

126 have 'failed' screening for authentic ancient DNA and are not included in the statistical

127 analyses. Additionally, individual 22 yielded relatively poor results for both treatments.

128 Only 533 reads (out of $\sim 4$ million unique reads sequenced) aligned to the 1.24 million

129 sites targeted in the nuclear genome for the MDE treatment, making it impossible to

130 calculate several of the reported metrics. While we did obtain enough reads $(23,239$ reads

131 out of $\sim 18$ million unique reads sequenced) for some analyses to produce results for the

132 tooth root that underwent Method WTR, the relatively low rate of mitochondrial match to

133 the consensus $(0.860)$ suggests that this sample is likely contaminated. Based on these

134 results, we also chose to exclude individual 22 from statistical analyses. However, we 
bioRxiv preprint doi: https://doi.org/10.1101/2020.08.19.256412; this version posted August 19, 2020. The copyright holder for this preprint (which was not certified by peer review) is the author/funder, who has granted bioRxiv a license to display the preprint in perpetuity. It is 18 ade available under aCC-BY-NC-ND 4.0 International license.

135 note that there are no significant changes to the reported statistics when the excluded

136 individuals are included in calculations for which metrics from both treatments are

137 available (Supplementary Table 2). For all statistical calculations, we included data from

138 all other samples, which were processed as either double-stranded (samples 1-10) or

139 single-stranded (samples 11-30) libraries. Results where each of these methods were

140 analyzed separately are reported in Supplementary Table 2.

\begin{tabular}{|c|c|c|c|c|c|c|c|c|c|c|c|c|}
\hline 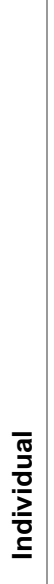 & 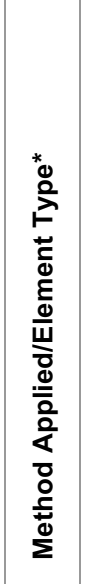 & 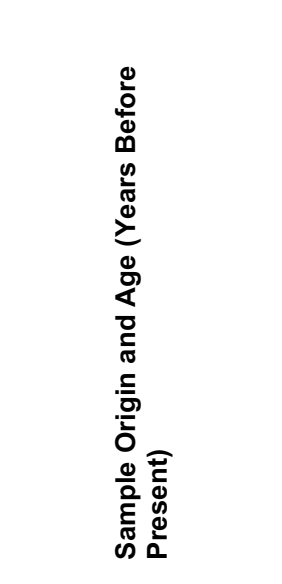 & 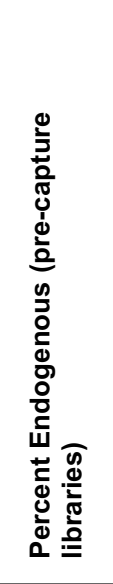 & 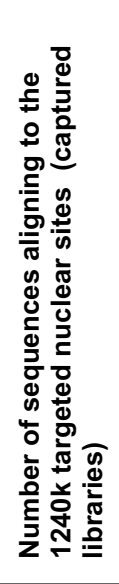 & 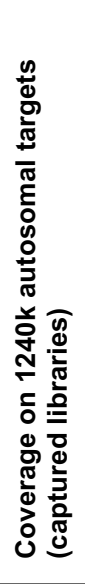 & 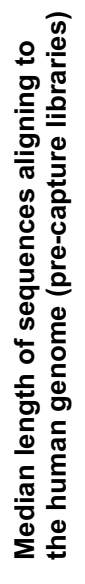 & 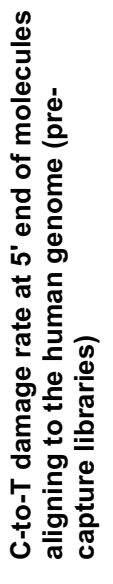 & 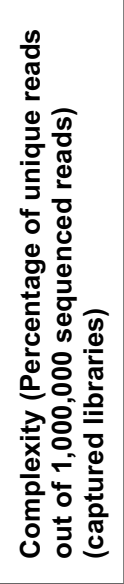 & 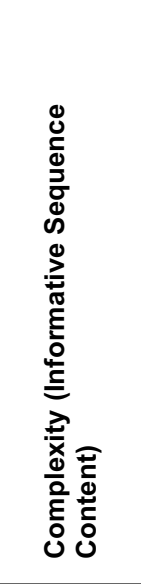 & 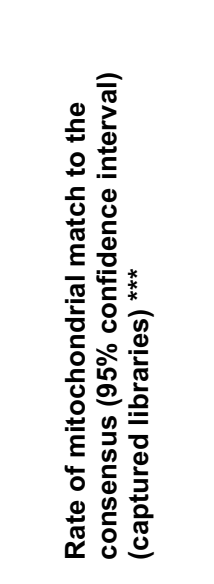 & 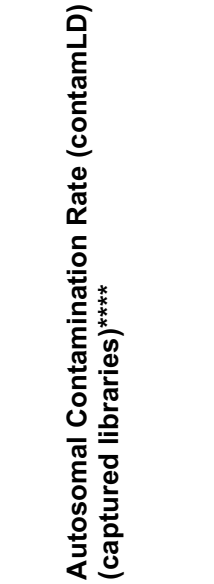 & 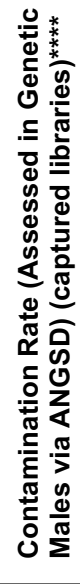 \\
\hline \multirow{3}{*}{1} & $P$ & \multirow{3}{*}{$\begin{array}{l}\text { Urziceni, Romania } \\
6,300-6,050 \text { BP }\end{array}$} & $68.23 \%$ & 732856 & 2.94 & 44 & 0.111 & $92.10 \%$ & $1.72 \mathrm{E}+10$ & $0.992+/-0.006$ & $-0.003+/-0.005$ & 0.007 \\
\hline & MDE & & $34.22 \%$ & 422192 & 0.37 & 48 & 0.072 & $30.50 \%$ & $1.12 E+09$ & $0.990+/-0.008$ & $-0.008+/-0.023$ & 0.025 \\
\hline & WTR & & $12.50 \%$ & 648760 & 0.88 & 46 & 0.055 & $51.80 \%$ & $8.74 \mathrm{E}+09$ & $0.997+/-0.004$ & $0.008+/-0.011$ & 0.006 \\
\hline \multirow{3}{*}{2} & $P$ & \multirow{3}{*}{$\begin{array}{l}\text { Urziceni, Romania } \\
6,300-6,050 \text { BP }\end{array}$} & $23.19 \%$ & 713660 & 2.78 & 44 & 0.107 & $91.20 \%$ & $2.93 \mathrm{E}+11$ & $0.986+/-0.010$ & $-0.01+/-0.006$ & 0.006 \\
\hline & MDE & & $8.51 \%$ & 507713 & 0.51 & 46 & 0.055 & $38.10 \%$ & $2.74 \mathrm{E}+09$ & $0.976+/-0.012$ & $-0.033+/-0.023$ & 0.003 \\
\hline & WTR & & $1.19 \%$ & 8438 & 3.32 & 50 & 0.045 & $0.90 \%$ & $2.03 E+06$ & $0.984+/-0.010$ & $-0.097+/-0.077$ & .. \\
\hline \multirow{2}{*}{$3^{* *}$} & MDE & \multirow{2}{*}{$\begin{array}{c}\text { Glăvăneşti, Romania } \\
5,450-3,050 \text { BP }\end{array}$} & $2.01 \%$ & 29234 & 0.02 & 39 & 0.102 & $2.80 \%$ & $1.24 \mathrm{E}+07$ & $0.803+/-0.087$ & .. & .. \\
\hline & WTR & & $0.65 \%$ & 62005 & 0.04 & 39 & 0.128 & $6.30 \%$ & $4.60 \mathrm{E}+08$ & $0.946+/-0.029$ & .. & .. \\
\hline \multirow{3}{*}{4} & $P$ & \multirow{3}{*}{$\begin{array}{c}\text { Glăvăneşti, Romania } \\
\text { 5,450-3,050 BP }\end{array}$} & $1.65 \%$ & 165145 & 0.12 & 39 & 0.147 & $14.30 \%$ & $9.78 \mathrm{E}+08$ & $0.936+/-0.024$ &.. & .. \\
\hline & MDE & & $72.77 \%$ & 624069 & 0.91 & 47 & 0.055 & $53.00 \%$ & $1.52 \mathrm{E}+10$ & $0.979+/-0.011$ & $-0.010+/-0.014$ & 0.010 \\
\hline & WTR & & $19.69 \%$ & 633735 & 0.87 & 47 & 0.047 & $52.30 \%$ & $5.73 \mathrm{E}+09$ & $0.993+/-0.005$ & $0.000+/-0.017$ & 0.002 \\
\hline \multirow{3}{*}{$5^{\star *}$} & $P$ & \multirow{3}{*}{$\begin{array}{c}\text { Ras al Hamra, Oman } \\
5,650-5,150 \mathrm{BP}\end{array}$} & $0.10 \%$ & 6226 & 0 & 60 & 0.000 & .. & $2.90 \mathrm{E}+06$ & . & .. &.. \\
\hline & MDE & & $2.74 \%$ & 8364 & 0.01 & 59 & 0.000 & $0.90 \%$ & $2.00 \mathrm{E}+06$ & .. & .. & .. \\
\hline & WTR & & $1.48 \%$ & 6655 & 0 & 61 & 0.000 & $0.70 \%$ & $2.15 \mathrm{E}+06$ & .. & .. & .. \\
\hline \multirow{3}{*}{$6^{* *}$} & $P$ & \multirow{3}{*}{$\begin{array}{c}\text { Ras al Hamra, Oman } \\
5,650-5,150 \mathrm{BP}\end{array}$} & $0.17 \%$ & 6530 & 0 & 58 & 0.000 & $0.70 \%$ & $3.61 \mathrm{E}+06$ & .. & ... & .. \\
\hline & MDE & & $0.97 \%$ & 8860 & 0.01 & 58 & 0.019 & $1.00 \%$ & $1.61 \mathrm{E}+06$ & .. & .. &.. \\
\hline & WTR & & $0.24 \%$ & 7846 & 0.01 & 58 & 0.029 & $0.90 \%$ & $2.84 \mathrm{E}+06$ & .. & .. & .. \\
\hline \multirow{3}{*}{7} & $\mathrm{P}$ & \multirow{3}{*}{$\begin{array}{c}\text { Cimișlia, Rep. of } \\
\text { Moldova } \\
2,050-1,850 \text { BP }\end{array}$} & $2.74 \%$ & 185208 & 0.15 & 38 & 0.282 & $16.40 \%$ & $6.36 \mathrm{E}+09$ & $0.988+/-0.009$ & $0.004+/-0.018$ & .. \\
\hline & MDE & & $57.34 \%$ & 486828 & 0.49 & 44 & 0.135 & $34.50 \%$ & $3.81 \mathrm{E}+09$ & $0.983+/-0.009$ & $0.022+/-0.009$ &.. \\
\hline & WTR & & $8.34 \%$ & 530939 & 0.58 & 45 & 0.064 & $39.80 \%$ & $4.50 \mathrm{E}+09$ & $0.993+/-0.006$ & $-0.013+/-0.011$ & .. \\
\hline \multirow{3}{*}{8} & $\mathrm{P}$ & \multirow{3}{*}{$\begin{array}{l}\text { Ciumai, Rep. of } \\
\text { Moldova } \\
4,000-1,000 \text { BP }\end{array}$} & $51.70 \%$ & 712417 & 2.76 & 44 & 0.149 & $90.20 \%$ & $2.73 E+11$ & $0.994+/-0.005$ & $-0.009+/-0.003$ & 0.004 \\
\hline & MDE & & $0.74 \%$ & 223292 & 0.17 & 45 & 0.077 & $19.00 \%$ & $3.92 E+08$ & $0.997+/-0.003$ & $-0.002+/-0.023$ & .. \\
\hline & WTR & & $31.64 \%$ & 683354 & 2.54 & 42 & 0.147 & $87.40 \%$ & $1.39 \mathrm{E}+11$ & $0.954+/-0.014$ & $-0.01+/-0.005$ & 0.007 \\
\hline 9 & $P$ & & $43.68 \%$ & 716356 & 2.53 & 45 & 0.130 & $86.70 \%$ & $1.73 \mathrm{E}+11$ & $0.989+/-0.007$ & $-0.003+/-0.006$ &.. \\
\hline
\end{tabular}


bioRxiv preprint doi: https://doi.org/10.1101/2020.08.19.256412; this version posted August 19, 2020. The copyright holder for this preprint (which was not certified by peer review) is the author/funder, who has granted bioRxiv a license to display the preprint in perpetuity. It is 9hade available under aCC-BY-NC-ND 4.0 International license.

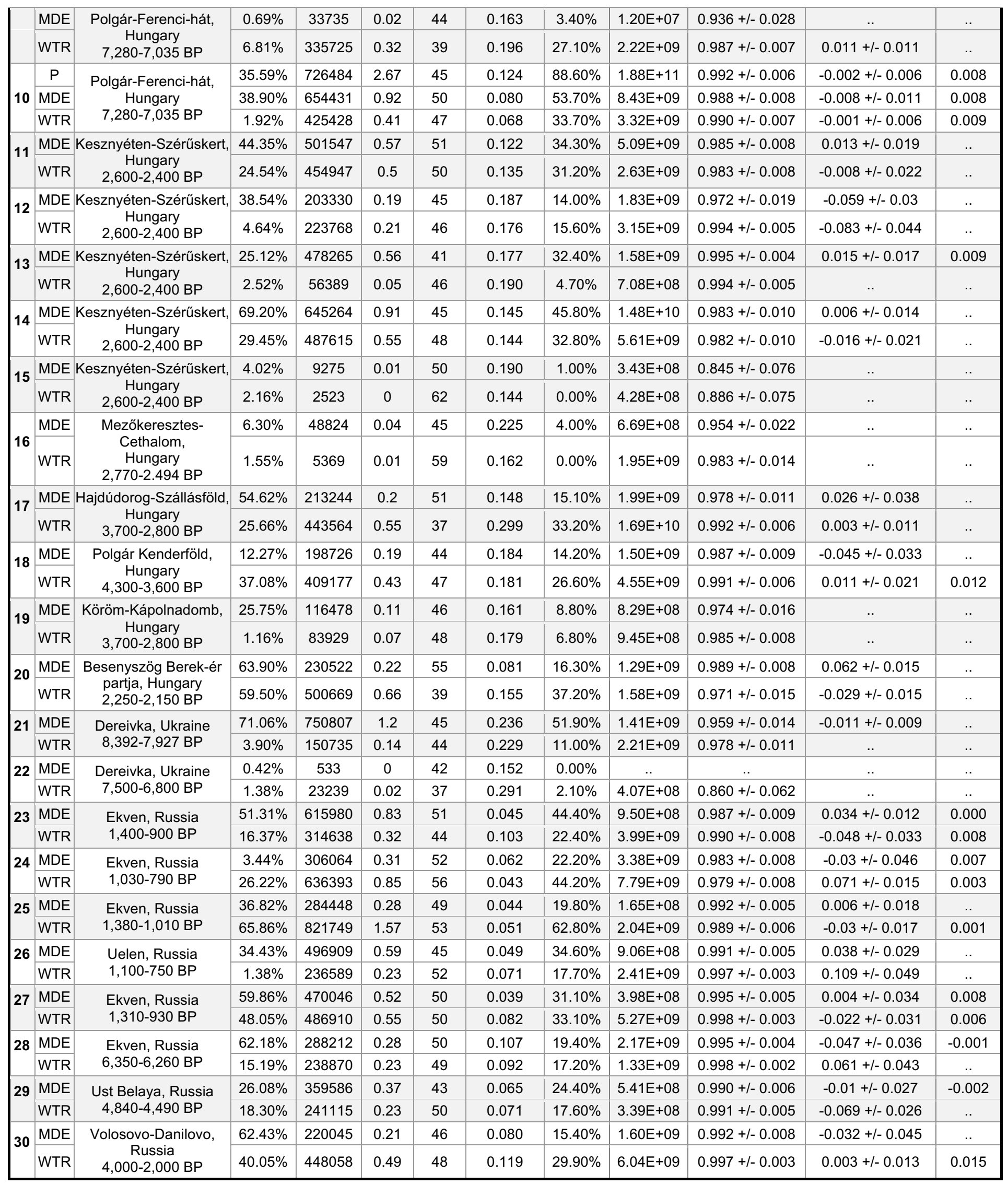


Table 1. Sample Information All estimates are made based on data produced from libraries that underwent the 1240k capture unless otherwise specified. *Sampling/Extraction Methods: P- Powdered Petrous Bone (Pinhasi et al. 2019), standard extraction (Dabney et al. 2013a); MDE- Tooth Root processed via Minimally Destructive Extraction; WTR- Whole Tooth Root, powdered with standard extraction (Dabney et al. 2013a). Extracts for individuals 1-10 were processed entirely manually and underwent partial UDG treatment followed by double stranded library preparation, while extracts for individuals 11-30 were processed robotically following incubation in extraction buffer (Rohland et al 2019, buffer D) and processed using USER treatment followed by single stranded library preparation.

${ }^{* *}$ Note that sample 3P was excluded from comparisons as it was determined bioinformatically that the petrous bone and tooth sampled did not originate from the same individual. Note also that the DNA preservation in samples 5 and 6 was too poor for further analysis. generate a contamination estimate based on either mitochondrial, autosomal or X-chromosome data. For X-chromosome based contamination estimates, ANGSD can only estimate contamination rates for individuals determined to be genetically male. Individuals who are female or for whom sex cannot be

157 determined (sex ND) are noted.

160 protocol immediately prior to extraction and 24 hours after extraction to allow for the

161 complete drying of the roots (Figure 1; Supplementary Figure 1). A slight degradation of

162 the outer tooth root surface is visible for many of the samples, as the portion of the tooth

164 to the unexposed portion. In the case of two of the most poorly preserved samples

165 (individuals 5 and 6), the tooth roots-one of which broke in two when cut from the tooth crown-crumbled during removal of the parafilm that covered the tops of the roots after

167 the incubation in extraction buffer. These results suggest that users should exercise 


\section{Complete tooth}
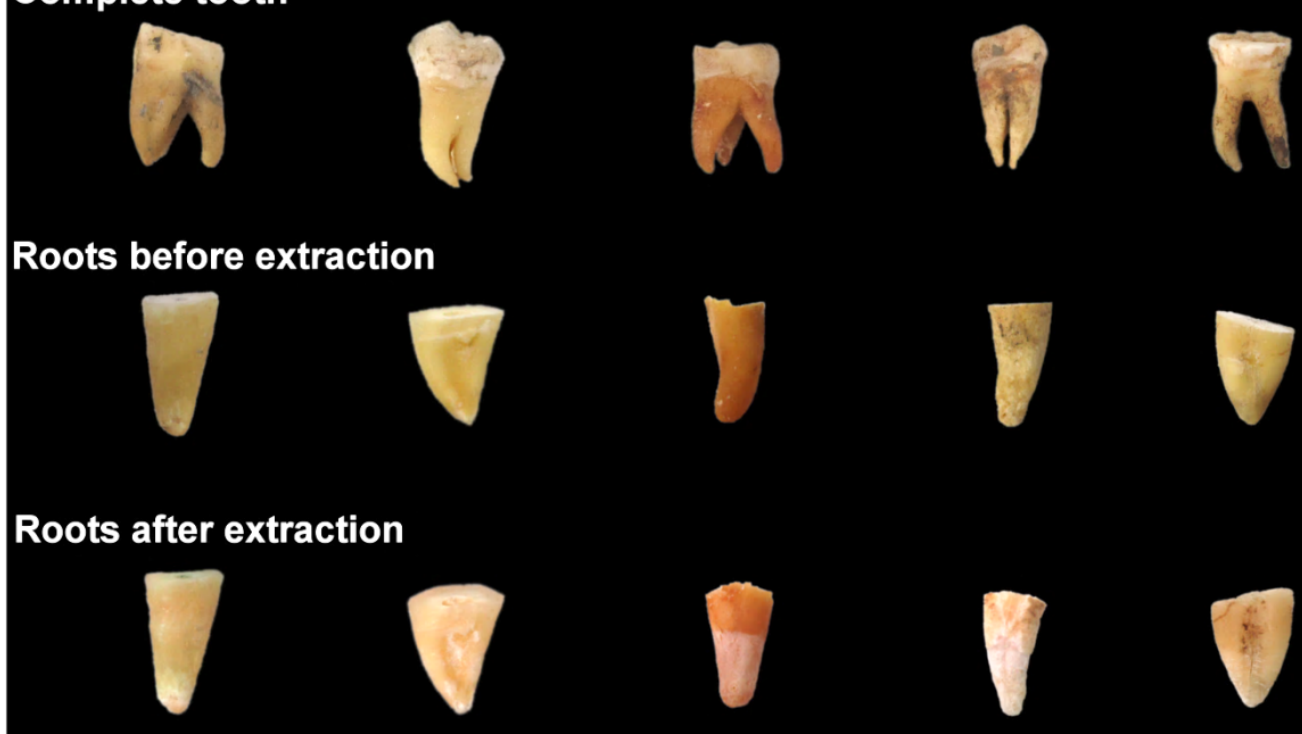

1

7

9

10

Figure 1. Tooth roots before and after minimally destructive extraction. The complete tooth is shown prior to processing (top). Tooth roots are shown immediately prior to extraction (middle) and 24 hours after extraction (bottom). See Supplementary Figure 1 for before and after images of all sampled teeth.

\section{Comparison of Minimally Destructive Extraction Protocol versus Powder-}

\section{Based Extraction Protocols}

Following bioinformatic processing, we generated summary statistics for each extract, including metrics of sample complexity and contamination rates (Table 1, Supplementary Table 1). In the following section, for each individual we compare the quality of ancient DNA yielded by the minimally destructive extraction method (Method MDE) to that produced by the destructive, traditional sampling methods (Methods WTR and P), using a Wilcoxon signed-rank test. The null hypothesis is that the difference between pairs of data generated using Method MDE and Method WTR or P follows a symmetric distribution around zero. The alternative hypothesis is that the difference between the paired data does not follow a symmetric distribution around zero. A threshold of $p$-value $=0.05$ is used 
186 to denote significance which can only be achieved if there are a minimum of 6

187 comparisons per test.

\section{Extraction Efficiency}

189 In order to assess the efficiency of the minimally destructive extraction method, we first

190 compare the proportion of endogenous molecules (i.e. molecules that align to the human

191 reference genome, hg19) in samples produced using each extraction method and

192 sequenced via shotgun (i.e. pre-capture) sequencing. While we observe a high degree of

193 variability (Figure 2a; Table 1) between treatment types for each individual, there is a

194 statistically significant difference in the proportion of endogenous molecules sequenced

195 using the MDE and WTR methods ( $p$-value=0.004), with an average of $35.8 \%$ and $18.8 \%$

196 endogenous molecules for each extraction method, respectively. These results support

197 previous assertions that the outer cementum layer of the tooth root, which is targeted by

198 the MDE method, contains a higher proportion of endogenous molecules than other

199 portions of the tooth root (Damgaard et al. 2015). In contrast, we do not observe a

200 significant difference in the proportion of endogenous molecules between methods MDE

201 and $P(p$-value=1.000) (Supplementary Figure 2a), with an average of $36.4 \%$ endogenous

202 observed when sampling from the petrous. These results are again consistent with claims

203 that the petrous and tooth cementum both contain relatively high proportions of 204 endogenous molecules (Damgaard et al. 2015; Hansen et al. 2017). While the high

205 proportion of endogenous molecules obtained using the MDE method is promising, 206 measuring the fraction of endogenous molecules in a sample does not tell us about the 207 total amount of DNA obtained using each method. 

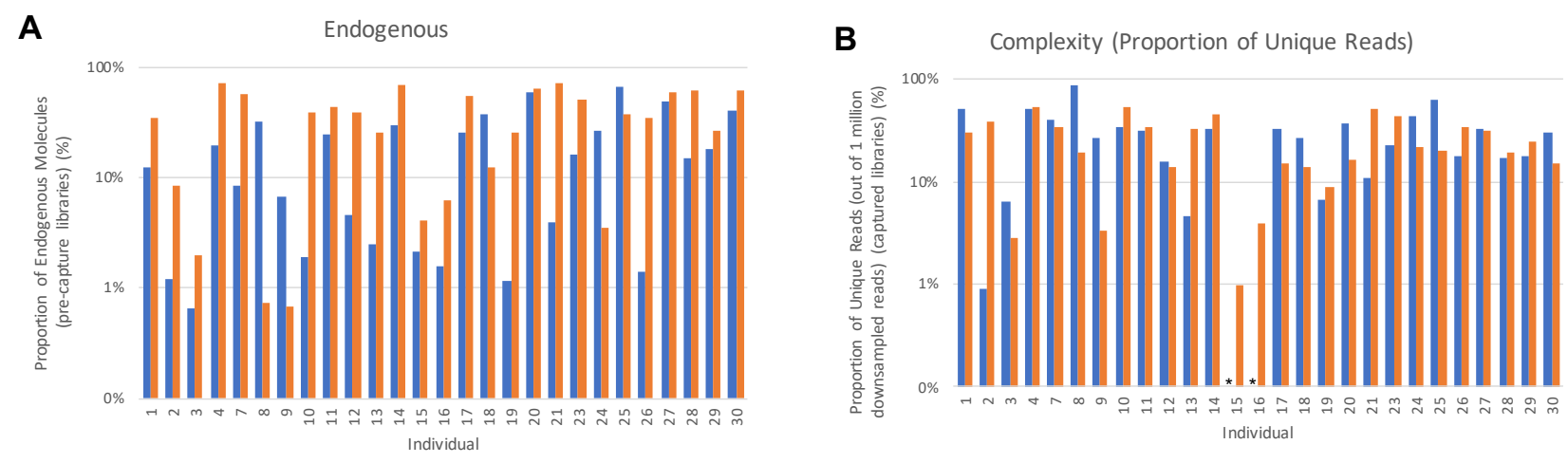

C

Complexity (Informative Sequence Content)
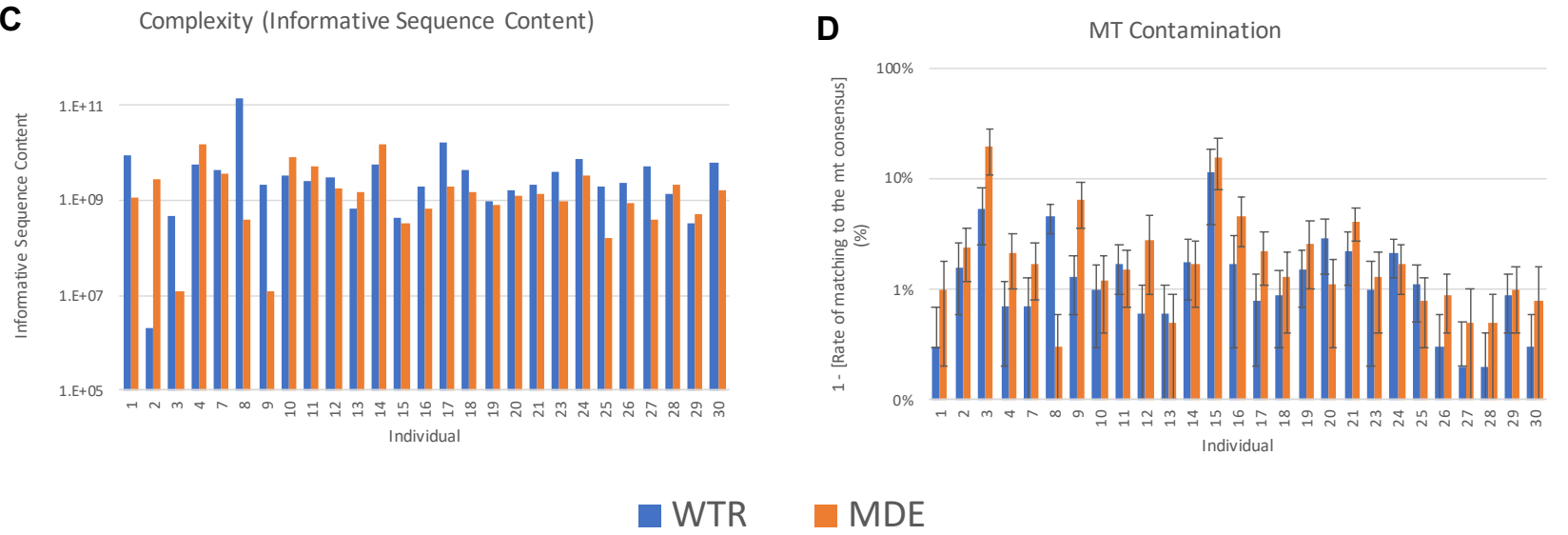

Figure 2: Sample Quality A comparison of the quality of data produced by WTR (Whole Tooth Root) and MDE (Minimally Destructive Extraction) Methods in samples that passed quality filtering. (A) The proportion of endogenous molecules in data obtained via shotgun sequencing. (B) The complexity of each sample, as measured by the proportion of unique reads out of $1,000,000$ reads sequenced. Asterisks indicate that the total number of unique reads sequenced was below $1,000,000$ for the specified sample, therefore complexity estimates could not be generated. (C) The complexity of each sample, as measured by informative sequence content (D) The rate of contamination is compared by considering the rate of matching to mitochondrial consensus sequence. Error bars indicate the $95 \%$ confidence interval. Only samples that passed quality screening are shown. Plots showing comparisons with samples generated using Method $\mathrm{P}$ are shown in Supplementary Figure 2. within a single library—using two metrics. In the first metric, we consider the proportion

210 of unique molecules sequenced in each sample, after down-sampling to $1,000,000$

211 sequences that align to the 1.24 million SNPs targeted during capture (Figure 2B). This

212 is a useful metric for comparison between samples, as it is not biased by differences in

213 sequencing depth across samples. However, as this metric is calculated using sequence

214 data for samples that underwent targeted enrichment capture, a process that may 
215 introduce bias into the data, we therefore also consider a second complexity metric, the

216 informative sequence content (Glocke and Meyer 2017). This metric quantifies the

217 relative proportion of molecules that were successfully amplified from each sample using

218 quantitative PCR (qPCR) analysis. The results are calibrated using the proportion of

219 endogenous molecules and average length of molecules measured in the shotgun

220 sequencing data, reflecting the number of sequences in the DNA extracts that can be

221 aligned to the human genome.

222

223 Neither complexity metric finds a statistically significant difference between complexity

224 measured in samples prepared using Method MDE versus Method WTR ( $p$-value=0.792

225 and 0.107 , for the first and second complexity metrics, respectively), suggesting that

226 using a minimally destructive extraction method does not result in loss of genetic data

227 when sampling from teeth (Figure 2B, Table 1). While we find no statistically significant

228 difference between samples prepared using Method MDE versus Method P using the first

229 complexity metric ( $p$-value=0.091), we do detect a significant difference using the second

230 metric ( $p$-value=0.043) (Supplementary Figure 2B-C). We note that the power of this

231 analyses is limited due to the low number of comparisons we were able to make $(\mathrm{N}=7)$,

232 therefore this comparison may warrant further study, particularly because previous

233 studies have found that the rates of ancient DNA preservation in cementum versus

234 petrous samples is dependent upon sample preservation (Hansen et al. 2017).

\section{Contamination Rate}

236 We were concerned that extracting ancient DNA directly from the outer layer of the tooth

237 root might result in a higher rate of contamination in the sample, especially due to the 
238 increased potential for exposure of this region to contaminants during handling. Standard 239 sampling protocols typically involve the physical removal of the outermost layer of bone

240 or tooth prior to sampling, using a sanding disc or a sandblaster, while, in contrast, the

241 minimally destructive extraction method specifically targets this outer layer following a

242 superficial chemical (bleach) and brief (5-10 minute) ultraviolet decontamination. We

243 therefore compare the relative contamination rates between sampling methods using a

244 variety of metrics. First, we compare the rate of matching to the mitochondrial consensus

245 sequence (Fu et al. 2013). A minimum threshold of $95 \%$ is typically applied during

246 screening of ancient DNA for population genetic studies. We observe substantial

247 variability in contamination rate between and within individuals for all treatment methods

248 (Figure 2D, Table 1). While we detect a significant difference between mitochondrial

249 match to consensus rates between the MDE and WTR methods ( $p$-value=0.004), the

250 average difference between these two methods is small ( $97.0 \%$ and $98.2 \%$, respectively).

251 Further, we observe no significant difference between the Methods MDE and $P(p=0.310)$

252 (Supplementary Figure 2D).

253

254 Next, we estimate the autosomal rate of contamination, using the tool ContamLD

255 (Nakatsuka et al. 2020), which measures the breakdown of linkage disequilibrium in a 256 sequenced individual, a process which is accelerated by increased contamination. We 257 again estimate relatively low rates of contamination across all samples, and find no 258 significant difference in contamination rates between Methods MDE and WTR ( $p$ 259 value $=0.490)$ or between Methods MDE and P ( $p$-value=0.893). 
261 We also estimate contamination rates in the individuals who are identified as genetically

262 male using ANGSD (Korneliussen et al. 2014). We obtain low estimates of contamination

$263(\leq 2.5 \%)$ across all male samples (Table 1). Comparing the X-chromosome contamination

264 estimates for the 6 genetically male individuals for whom there was enough data to

265 produce estimates for both treatment types, we do not detect a significant difference

266 between the MDE and WTR Methods ( $p$-value=0.293). Taken together these three

267 estimates of contamination suggest that, in practice, the UV and bleach decontamination

268 protocol used for the MDE Method performs similarly to the physical surface removal

269 decontamination steps implemented in the destructive protocols, and is sufficient to

270 produce ancient DNA data of analyzable quality.

272 We considered the read length distribution and frequency of C-to-T damage in the 273 terminal bases of reads that aligned to the human genome (hg19) that were obtained via

274 shotgun sequencing (i.e. pre-capture). Authentic ancient DNA is thought to consist of 275 characteristically short fragments, with very few reads longer than 100 base pairs (Sawyer 276 et al. 2012; Dabney et al. 2013b; Glocke and Meyer 2017), therefore the read length 277 distribution is used as a general metric to assess ancient DNA authenticity. We find that 278 all samples appear to have read length profiles characteristic for authentic ancient DNA 279 (Supplementary Figure 3) and we do not observe a significant difference in median length 280 of reads obtained using Method MDE and Method WTR ( $p$-value=0.375). A weakly 281 significant difference is observed between reads obtained using Method MDE and P ( $p-$ 
282 value $=0.034)($ Table 1$)$, suggesting that there may be systematic differences between

283 DNA preservation in petrous and tooth samples.

284

285 Endogenous ancient DNA samples are also thought to exhibit a high rate of C-to-T 286 damage, particularly in the terminal bases. Using a partial or USER UDG treatment for 287 double stranded and single stranded libraries, respectively (Rohland et al. 2015; 288 Gansauge et al. in Prep), we removed this damage in the interior of each molecule, while 289 retaining it in the terminal bases. Therefore, we are able to use the frequency of these 290 errors to assess ancient DNA authenticity. For samples processed using Method MDE 291 and WTR ( $p$-value=0.249) we observe no significant difference in the frequencies of C292 to-T damage in terminal bases at the 5 ' end of molecules that aligned to the human 293 genome (hg19), obtained via shotgun sequencing. However, the distribution of damage 294 rates in samples processed using Method P are significantly different to Method MDE (p295 value=0.028), with higher rates of damage observed in libraries produced using Method 296 P in most (8/9) cases, again suggesting that there may be systematic differences between 297 DNA preservation in petrous and tooth samples (Table 1, Supplementary Figure 4 \& 5).

299 Finally, we were concerned that the use of parafilm to cover portions of the tooth roots 300 that we did not want expose to the extraction buffer could serve as a possible source of 301 contamination. We therefore created a parafilm extraction control, in which a small strip 302 of parafilm (comparable in size to that used for covering the tooth roots), was added to a 303 tube of extraction buffer and underwent sample processing along with the MDE samples 304 and regular extraction blanks. We observe very few reads associated with this parafilm 
305 blank (Supplementary Table 1), suggesting that the use of parafilm does not serve as a

306 significant source of contamination in the MDE Method.

\section{DISCUSSION}

308 This minimally destructive sampling protocol enables extraction of ancient DNA from the

309 cementum portion of tooth roots that is of similar quality to ancient DNA obtained from

310 teeth using traditional, destructive sampling methods that rely on powder produced

311 through drilling or cutting and powdering. This is true with regards to both the amount of

312 DNA that it is possible to obtain and the levels of contamination detected in the samples.

313 In contrast, our results suggest that DNA sampled from the petrous bone exhibits more

314 complexity than DNA sampled from the tooth cementum, indicating that there is still

315 justification for choosing to sample from petrous bones over teeth when trying to

316 maximize the chances of successfully sequencing ancient DNA, particularly in cases

317 where sample preservation is poor-a circumstance in which ancient DNA sampled from

318 petrous has previously been found to be of higher quality than in cementum (Hansen et

319 al. 2017). However, the physical damage to the sampled tooth is substantially reduced

320 and the morphological integrity of the sampled tooth is retained when using this minimally

321 destructive sampling protocol, making this an optimal sampling method of teeth in cases

322 where sample preservation is of the highest priority.

324 One of the major concerns surrounding an extraction protocol that targets the outer

325 surface of an ancient sample is the potential for an increase in contamination, as this

326 outer surface may come in direct contact with various contaminants, particularly during 
327 handling. Since the majority of samples selected for ancient DNA analysis have been excavated and manipulated without any consideration for potential future genetic studies,

329 this is of particular concern. While destructive methods physically remove the outermost

330 layer of bones and teeth to reduce contamination, we instead applied a bleach and UV

331 decontamination procedure to the tooth before processing. We detected little difference

332 in contamination rates between samples processed using this minimally destructive

333 decontamination and sampling method and those processed using standard destructive

334 methods. Further, these results suggest that decontamination procedures that involve

335 wiping a sample with bleach do not significantly reduce DNA yields, as opposed to

336 previously proposed decontamination methods involving the soaking of the sample for an

337 extended period of time (e.g. Higgins et al. 2013). By targeting the outer cementum tooth

338 surface directly, this method maximizes the proportion of cementum matrix which is being

339 digested and minimizes the amount of dentine sampled when compared to other 340 cementum-targeting methods (Damgaard et al. 2015), which sample a significant

341 proportion of the inner dentine layer in addition to the cementum. Furthermore, we find

342 that parafilm can be used to protect portions of the tooth that users do not wish to sample

343 (i.e. the tooth crown) from exposure to extraction buffer, without increasing contamination

344 rates.

346 While these results show that this minimally destructive approach is a promising

347 alternative to destructive sampling methods that are traditionally applied to ancient teeth,

348 we stress that further research is needed to determine whether it is recommended to opt

349 for this sampling method in all circumstances. Particularly, we note that the majority of 
350 teeth chosen for this analysis were of moderate to excellent preservation status. The two

351 most poorly preserved individuals included in this study contained too little DNA to allow

352 for comparisons to be made between Methods MDE and WTR, and the tooth roots

353 processed via Method MDE sustained damage during processing. Further study of the

354 utility of this method on less well-preserved teeth is therefore of great interest.

356 As the impact on dental morphology is minimal, this approach enables the preservation

357 of samples for future analyses. Previous studies have shown that exposure to the 358 chemicals used for ancient DNA extraction (mainly EDTA and proteinase K) do not affect

359 a specimen's suitability for subsequent biochemical analyses, such as radiocarbon (AMS

360 C14) dating (Korlević et al. 2018). Therefore, teeth processed using this minimally

361 destructive protocol would remain suitable for future biochemical analyses.

363 This minimally destructive extraction method drastically reduces the amount of physical 364 destruction caused by ancient DNA extraction, creating no holes or cuts in the sampled 365 tooth or bone, while also shortening the overall length of the extraction protocol, without 366 meaningfully increasing the amount of contamination. This method makes it possible to 367 extract ancient DNA from individuals that would otherwise be unavailable for ancient DNA 368 study due to the destructive nature of traditional sampling methods.

\section{METHODS}

370 All ancient DNA analyses were performed in dedicated clean rooms at the University of

371 Vienna and Harvard Medical School. For individuals 1-10, skeletal sampling, preparation 
372 and DNA extraction were performed at the University of Vienna. Library preparation,

373 targeted enrichment capture, and sequencing was performed at Harvard Medical School.

374 For individuals 11-30, skeletal sampling was performed at the University of Vienna, while

375 all other processing was performed at Harvard Medical School.

376 Sampling

377 We selected skeletal elements from 30 ancient individuals of varying age, geographic 378 origin, and degree of preservation for analysis (Table 1). From each individual, we

379 selected a single multi-rooted tooth for sampling. For the first 10 individuals, we also 380 selected a temporal bone for sampling. We UV irradiated each tooth in a cross-linker for

3815 to 10 minutes on each side, in order to remove as much surface contamination as

382 possible. We then cut off the roots of each tooth using a diamond cutting disc and a hand-

383 held Dremel drill, treating each root separately in all subsequent analyses. From each

384 individual, we randomly selected one tooth root ("Method MDE") for minimally destructive 385 extraction. These tooth roots were subject to additional surface cleaning by wiping the 386 teeth clean with a $2 \%$ bleach solution and rinsing with $95 \%$ ethanol, followed by UV387 irradiation for 5 to 10 minutes on each side. We prepared the second set of tooth roots 388 ("Method WTR") by removing the extreme outer surface of each tooth root using a sanding 389 disc and drill, and milling the root in a Retsch MM400 mixer mill for a total of 60 seconds 390 with a 10 seconds break after 30 seconds to produce a powder. Additionally, we obtained 391 approximately 50mg of bone powder from the petrous portion of each of the 10 selected 392 temporal bones, using standard methods ("Method P") (Pinhasi et al. 2019). 


\section{DNA Extraction}

394 We prepared selected tooth roots (Method MDE) for minimally destructive extraction by

395 recording the initial weight of the tooth root, then isolating the targeted portion of the tooth

396 root using parafilm (Supplementary Figure 6; see Supplementary Information 1 for a step-

397 by-step description of the minimally destructive extraction method). We targeted the lower

398 portion of the tooth root, where cellular cementum is concentrated. All other surfaces were

399 wrapped in UV-decontaminated parafilm in order to prevent significant contact with the

400 extraction buffer. The tooth roots were placed in $750 \mu \mathrm{L}-1 \mathrm{~mL}$ of extraction buffer $(0.45$

401 M EDTA, 0.25 mg/mL Proteinase K, pH 8.0; defined in Rohland and Hofreiter (2007) with

402 the exposed portion pointing down, and incubated for 2.5 hours at $37^{\circ} \mathrm{C}$, shaking gently.

403 Following incubation, the roots were removed from the extraction buffer, which was then

404 processed according to standard ancient DNA extraction procedures. Samples from 405 individuals 1-10 underwent manual ancient DNA extraction, as described in Dabney et al.

406 (2013a), with modifications. The MinElute columns were replaced with a preassembled 407 spin column device (Roche, as described in Korlević et al. (2015)). We washed lysates 408 with $650 \mu \mathrm{L}$ of PE buffer (Qiagen) and spun at 6000 rpm for 1 minute. Following dry spin, 409 we isolated the DNA by placing the spin column in a fresh $1.5 \mathrm{~mL}$ collection tube, and 25 $410 \mu \mathrm{L}$ TET buffer was pipetted onto the column's silica membrane, which was incubated at

411 room temperature for 10 minutes, and then spun at maximum speed for 30 seconds. We 412 repeated this step, producing a total of $50 \mathrm{uL}$ of DNA extract. Samples from individuals 413 11-30 underwent robotic extraction following incubation, using the robotic protocol 414 described in Rohland et al. (2018), using buffer D. 
416 For samples processed using Methods WTR and P, sampled bone powders were

417 incubated overnight ( 18 hours) in extraction buffer at $37^{\circ} \mathrm{C}$, with gentle shaking. For

418 samples from individuals $1-10$, up to $50 \mathrm{mg}$ bone powder was incubated in $1 \mathrm{~mL}$ extraction

419 buffer, which then underwent manual extraction, as described above. For samples from

420 individuals 11-30, 37 mg of bone powder was incubated in $750 \mu \mathrm{L}$ extraction buffer, and

421 then underwent robotic extraction, as described above.

423 Negative controls were prepared alongside ancient DNA extracts for all extraction

424 batches. In each case, extraction buffer was added to an empty tube prior to incubation,

425 and the negative control was treated identically to all other samples during subsequent

426 processing. Additionally, we generated one parafilm extraction control, by incubating a

427 piece of UV-decontaminated parafilm in extraction buffer overnight in order to determine

428 whether the parafilm coverings used to protect the ends of the tooth roots might be a

429 potential source of contamination.

431 Following incubation in the extraction buffer, the roots were rinsed with $95 \%$ ethanol in

432 order to remove any remaining extraction buffer and air dried at room temperature for 24

433 hours. The samples were then re-weighed to assess the total amount of dental material

434 digested.

435 Library Preparation, Enrichment, and Sequencing

436 We prepared double-stranded (samples 1-10) or single-stranded (samples 11-30)

437 libraries from $10 \mu \mathrm{L}$ of each extract using UDG-treatment methods, as described in 438 Rohland et al. (2015) and Gansauge et al. (in Prep), respectively. These methods remove 
439 ancient DNA damage at the interior of each DNA sequence, while preserving 440 characteristic ancient DNA damage at the terminal ends of the molecules, to be used for

441 ancient DNA authentication during bioinformatic processing. We enriched libraries for

442 human DNA via targeted enrichment at 1.24 million SNP sites that are informative for

443 population genetic analyses (Fu et al. 2015; Haak et al. 2015; Mathieson et al. 2015).

444 Following enrichment, libraries were sequenced on an Illumina NextSeq500 machine, 445 with $2 \times 76$ or $2 \times 101$ cycles, with an additional $2 \times 7$ or $2 \times 8$ cycles used for identification of 446 indices, for double-stranded and single-stranded libraries, respectively.

\section{Bioinformatic Processing}

448 We trimmed molecular adapters and barcodes from sequenced reads, and the merged 449 paired end reads, requiring an overlap of 15 base pairs (allowing up to three mismatches 450 of low base quality $(<20)$ or one mismatch of high base quality $(\geq 20)$ ) using custom 451 software (https://github.com/DReichLab/ADNA-Tools). We then aligned the merged 452 sequences to both the mitochondrial RSRS genome (Behar et al. 2012) and the hg19 453 human reference sequence using samse in bwa (v0.6.1) (Li and Durbin 2009). We 454 identified duplicate reads, defined as having the same start and end position and 455 orientation, and a shared DNA barcode (unique quadruple barcode combinations are 456 inserted during library preparation), and retained only the copy with the highest quality 457 sequence.

459 We assessed ancient DNA authenticity using several metrics. We used the tool 460 ContamMix (Fu et al. 2014) to determine the rate of matching between mitochondrial 461 reads and the consensus sequence. The tool ContamLD was used to estimate the rate 
462 of contamination in the autosomes, based on the degree of breakdown of linkage 463 disequilibrium observed in each library relative to a panel of representative individuals

464 from the 1000 Genomes project (Nakatsuka et al. 2020). We determined the amount of 465 contamination in the $\mathrm{X}$-chromosome for male individuals using the tool ANGSD 466 (Korneliussen et al. 2014). Finally, we estimated the rate of C-to-T substitution at the

467 terminal ends of molecules for each sample (Jónsson et al. 2013) and the lengths of 468 sequenced molecules were considered as metrics of DNA authenticity for each sample.

470 We assessed the quality of ancient DNA observed by measuring the percent of 471 endogenous (unique reads that align to the human genome), coverage (average number 472 of reads aligning to each of the 1.24 million targeted SNP sites), and overall complexity 473 of the sample - assessed by determining the proportion of unique reads sequenced, after 474 randomly down-sampling to $1,000,000$ on-target reads, or by measuring the informative 475 sequence content (Glocke and Meyer 2017), in order to minimize bias caused by 476 differences in sequencing depth. 


\section{ACKNOWLEDGEMENTS}

We thank Iñigo Olalde and Nathan Nakatsuka for contributions to the bioinformatic analyses. E.H. was supported by a graduate student fellowship from the Max PlanckHarvard Research Center for the Archaeoscience of the Ancient Mediterranean (MHAAM). D.R. is an Investigator of the Howard Hughes Medical Institute and this work was also supported by John Templeton Foundation grant 61220 . Tamás Hajdu, Tamás Szeniczey, János Dani and Krisztián Kiss were supported by grant from the Hungarian Research, Development and Innovation Office, project number: FK128013. Alexandra Anders was supported by a grant from the Hungarian National Research, Development and Innovation Fund (Grant K124326).

\section{DISCLOSURE DECLARATION}

The authors declare no conflicts of interests.

\section{REFERENCES}

Behar DM, van Oven M, Rosset S, Metspalu M, Loogväli E-L, Silva NM, Kivisild T, Torroni A, Villems R. 2012. A "Copernican" reassessment of the human mitochondrial DNA tree from its root. The American Journal of Human Genetics 90: 675-684.

Bell LS, Kayser M, Jones C. 2008. The mineralized osteocyte: a living fossil. American Journal of Physical Anthropology: The Official Publication of the American Association of Physical Anthropologists 137: 449-456.

Bolnick DA, Bonine HM, Mata-Míguez J, Kemp BM, Snow MH, LeBlanc SA. 2012. Nondestructive sampling of human skeletal remains yields ancient nuclear and mitochondrial DNA. American Journal of Physical Anthropology 147: 293-300.

Bosshardt DD, Selvig KA. 1997. Dental cementum: the dynamic tissue covering of the root. Periodontology 2000 13: 41-75.

Carpenter ML, Buenrostro JD, Valdiosera C, Schroeder H, Allentoft ME, Sikora M, Rasmussen M, Gravel S, Guillén S, Nekhrizov G. 2013. Pulling out the 1\%: wholegenome capture for the targeted enrichment of ancient DNA sequencing libraries. The American Journal of Human Genetics 93: 852-864.

Correa H, Carneiro L, Yoshitake N, Carneiro A, Bizo G. 2019. Powder-Free DNA Extraction from Post-Mortem Teeth. J Forensic Res 10: 2.

Dabney J, Knapp M, Glocke I, Gansauge M-T, Weihmann A, Nickel B, Valdiosera C, García N, Pääbo S, Arsuaga J-L. 2013a. Complete mitochondrial genome sequence of a Middle Pleistocene cave bear reconstructed from ultrashort DNA fragments. Proceedings of the National Academy of Sciences 110: 15758-15763.

Dabney J, Meyer M, Pääbo S. 2013b. Ancient DNA damage. Cold Spring Harbor perspectives in biology 5: a012567.

Damgaard PB, Margaryan A, Schroeder H, Orlando L, Willerslev E, Allentoft ME. 2015. Improving access to endogenous DNA in ancient bones and teeth. Scientific reports 5: 11184. 
de León MSP, Koesbardiati T, Weissmann JD, Milella M, Reyna-Blanco CS, Suwa G, Kondo O, Malaspinas A-S, White TD, Zollikofer CP. 2018. Human bony labyrinth is an indicator of population history and dispersal from Africa. Proceedings of the National Academy of Sciences 115: 4128-4133.

Freeman E. 1994. Oral histology: development, structure, and function. Periodontium Mosby, St Louis: 276-312.

Fu Q, Hajdinjak M, Moldovan OT, Constantin S, Mallick S, Skoglund P, Patterson N, Rohland N, Lazaridis I, Nickel B. 2015. An early modern human from Romania with a recent Neanderthal ancestor. Nature 524: 216-219.

Fu Q, Li H, Moorjani P, Jay F, Slepchenko SM, Bondarev AA, Johnson PL, Aximu-Petri A, Prüfer K, de Filippo C. 2014. Genome sequence of a 45,000-year-old modern human from western Siberia. Nature 514: 445-449.

Fu Q, Meyer M, Gao X, Stenzel U, Burbano HA, Kelso J, Pääbo S. 2013. DNA analysis of an early modern human from Tianyuan Cave, China. Proceedings of the National Academy of Sciences 110: 2223-2227.

Gamba C, Jones ER, Teasdale MD, McLaughlin RL, Gonzalez-Fortes G, Mattiangeli V, Domboróczki L, Kővári I, Pap I, Anders A. 2014. Genome flux and stasis in a five millennium transect of European prehistory. Nature communications 5: 5257.

Gansauge M, Aximu-Petri A, Nagel S, Meyer M. in Prep. Manual and automated preparation of single-stranded DNA libraries for the sequencing of DNA from ancient biological remains and other sources of highly degraded DNA

Glocke I, Meyer M. 2017. Extending the spectrum of DNA sequences retrieved from ancient bones and teeth. Genome research 27: 1230-1237.

Grzesik WJ, Cheng H, Oh JS, Kuznetsov SA, Mankani MH, Uzawa K, Robey PG, Yamauchi M. 2000. Cementum-forming cells are phenotypically distinct from boneforming cells. Journal of Bone and Mineral Research 15: 52-59.

Haak W, Lazaridis I, Patterson N, Rohland N, Mallick S, Llamas B, Brandt G, Nordenfelt S, Harney E, Stewardson K. 2015. Massive migration from the steppe was a source for Indo-European languages in Europe. Nature 522: 207-211.

Hansen HB, Damgaard PB, Margaryan A, Stenderup J, Lynnerup N, Willerslev E, Allentoft ME. 2017. Comparing ancient DNA preservation in petrous bone and tooth cementum. PloS one 12.

Higgins D, Austin JJ. 2013. Teeth as a source of DNA for forensic identification of human remains: a review. Science \& Justice 53: 433-441.

Higgins D, Kaidonis J, Townsend G, Hughes T, Austin JJ. 2013. Targeted sampling of cementum for recovery of nuclear DNA from human teeth and the impact of common decontamination measures. Investigative genetics 4: 18.

Jónsson H, Ginolhac A, Schubert M, Johnson PL, Orlando L. 2013. mapDamage2. 0: fast approximate Bayesian estimates of ancient DNA damage parameters. Bioinformatics 29: 1682-1684.

Korlević P, Gerber T, Gansauge M-T, Hajdinjak M, Nagel S, Ayinuer-Petri A, Meyer M. 2015. Reducing microbial and human contamination in DNA extractions from ancient bones and teeth. BioTechniques 59: 87-93.

Korlević P, Talamo S, Meyer M. 2018. A combined method for DNA analysis and radiocarbon dating from a single sample. Scientific reports 8: 1-10. 
Korneliussen TS, Albrechtsen A, Nielsen R. 2014. ANGSD: analysis of next generation sequencing data. BMC bioinformatics 15: 356.

Li H, Durbin R. 2009. Fast and accurate short read alignment with Burrows-Wheeler transform. Bioinformatics 25: 1754-1760.

Makarewicz C, Marom N, Bar-Oz G. 2017. Palaeobiology: Ensure equal access to ancient DNA. Nature 548: 158.

Mathieson I, Lazaridis I, Rohland N, Mallick S, Patterson N, Roodenberg SA, Harney E, Stewardson K, Fernandes D, Novak M. 2015. Genome-wide patterns of selection in 230 ancient Eurasians. Nature 528: 499-512.

Nakatsuka NJ, Harney E, Mallick S, Mah M, Patterson N, Reich DE. 2020. ContamLD: Estimation of Ancient Nuclear DNA Contamination Using Breakdown of Linkage Disequilibrium. bioRxiv.

Pinhasi R, Fernandes D, Sirak K, Novak M, Connell S, Alpaslan-Roodenberg S, Gerritsen F, Moiseyev V, Gromov A, Raczky P et al. 2015. Optimal Ancient DNA Yields from the Inner Ear Part of the Human Petrous Bone. PLOS ONE 10: e0129102.

Pinhasi R, Fernandes DM, Sirak K, Cheronet O. 2019. Isolating the human cochlea to generate bone powder for ancient DNA analysis. Nature protocols: 1.

Prendergast ME, Sawchuk E. 2018. Boots on the ground in Africa's ancient DNA 'revolution': archaeological perspectives on ethics and best practices. antiquity 92: 803-815.

Rohland N, Glocke I, Aximu-Petri A, Meyer M. 2018. Extraction of highly degraded DNA from ancient bones, teeth and sediments for high-throughput sequencing. Nature Protocols: 1.

Rohland N, Harney E, Mallick S, Nordenfelt S, Reich D. 2015. Partial uracil-DNAglycosylase treatment for screening of ancient DNA. Phil Trans $R$ Soc $B$ 370: 20130624.

Rohland N, Hofreiter M. 2007. Ancient DNA extraction from bones and teeth. Nature protocols 2: 1756.

Rohland N, Siedel H, Hofreiter M. 2004. Nondestructive DNA extraction method for mitochondrial DNA analyses of museum specimens. Biotechniques 36: 814-821.

Sawyer S, Krause J, Guschanski K, Savolainen V, Pääbo S. 2012. Temporal patterns of nucleotide misincorporations and DNA fragmentation in ancient DNA. PloS one 7: e34131.

Sirak K, Fernandes D, Cheronet O, Harney E, Mah M, Mallick S, Rohland N, Adamski N, Broomandkhoshbacht N, Callan K. 2020. Human auditory ossicles as an alternative optimal source of ancient DNA. Genome Research 30: 427-436.

Sirak KA, Fernandes DM, Cheronet O, Novak M, Gamarra B, Balassa T, Bernert Z, Cséki A, Dani J, Gallina JZ. 2017. A minimally-invasive method for sampling human petrous bones from the cranial base for ancient DNA analysis. BioTechniques 62: 283-289.

Sirak KA, Sedig JW. 2019. Balancing analytical goals and anthropological stewardship in the midst of the paleogenomics revolution. World Archaeology: 1-14.

Slatkin M, Racimo F. 2016. Ancient DNA and human history. Proceedings of the National Academy of Sciences 113: 6380-6387.

Zhao N, Foster B, Bonewald L. 2016. The cementocyte-an osteocyte relative? Journal of dental research 95: 734-741. 